\title{
Quintessential Inflation and Non-Linear Effects of the Tachyonic Trap Mechanism
}

\author{
Mindaugas Karčiauskas and Stanislav Rusak \\ Departamento de Física Teórica and Instituto de Física de Partículas y del Cosmos IPARCOS and \\ Universidad Complutense de Madrid, E-28040 Madrid, Spain \\ Alejandro Saez \\ Department of Theoretical Physics, Universidad Autónoma de Madrid, 28049 Madrid, Spain and \\ Instituto de Física Teórica UAM-CSIC, c/ Nicolás Cabrera 13-15, \\ Universidad Autónoma de Madrid, 28049 Madrid, Spain
}

\begin{abstract}
With the help of the tachyonic trapping mechanism one can potentially solve a number of problems affecting quintessential inflation models. In this mechanism we introduce a trapping field with a spontaneous symmetry breaking potential. When the quintessential inflaton passes the critical point, a sudden burst of particle production is able to reheat the universe and trap the inflaton away from the minimum of its potential. However, self-interactions of the trapping field suppress particle production and reduce the efficiency of this process. We develop a method to compute the magnitude of the suppression and explore the parameter space in which the mechanism can be applied effectively.
\end{abstract}

\section{INTRODUCTION}

The origin of the accelerated expansion of the Universe remains one of the biggest puzzles in cosmology. The most direct explanation of such an expansion is the cosmological constant [1]. Such an explanation is also perfectly consistent with current observations [2]. Unfortunately, the cosmological constant explanation suffers from serious theoretical issues. Specifically, it is difficult to find a theoretical justification for its extremely small value.

It is thought that explaining the absence of the vacuum energy by invoking some unknown symmetry should be easier than explaining its tiny value required to fit the observations. If that is true, the current accelerated expansion could be driven by the potential energy of some slow rolling scalar field, called quintessence [3 8 . This mechanism is inspired by cosmic inflation and shares some of its features. Going one step further, it is argued that unifying the inflaton and quintessence into one and the same field has additional benefits. Such models are called quintessential inflation [9-11] (see ref. [12-15] for more recent reviews and references). Apart from being economic in its field content, another benefit of such models is that inflation provides initial conditions for the quintessential phase of the evolution. Otherwise initial conditions are free parameters.

Although, the quintessence and quintessential inflation were introduced to explain the apparent fine tuning of the cosmological constant, they bring another set of "tunings". Many of those stem from the fact that the scalar field has to be slowly rolling down the potential to provide dark energy. This can be achieved if the dynamics of quintessence is determined by the Hubble friction, which requires that the effective mass of the field is much smaller that the Hubble parameter today, $m_{\varphi} \equiv \sqrt{V^{\prime \prime}(\varphi) / 2}<H_{0}$, where $H_{0} \simeq 10^{-33} \mathrm{eV}$. A scalar field with such a tiny mass is problematic form the observational as well as theoretical points of view. On the one hand, a generic scalar field with such a small mass should have been detected, as it leads to several observable phenomena related to the 5th force in Nature 16. From the theoretical point of view, it is difficult to explain such a tiny mass within the framework of effective field theories [17].

An additional challenge for quintessential inflation models are super-Planckian field values. In order to explain inflation as well as the dark energy, which require hugely different energy scales, the potential must have a very large gradient. Due to such a gradient the $\varphi$ field picks up very large kinetic energy after inflation. The latter is difficult to dissipate before the field reaches super-Planckian values. This can be problematic within the effective field theory approach as one can find it complicated to justify the absence of non-renormalisable terms in the action [17] (models based on $\alpha$-attractors may avoid this problem, see for example ref. [18 20]).

In this work we investigate a model first proposed in ref. [21, which could address the above mentioned issues. In that work it was suggested that the quintessence field $\varphi$ interacts with another field $\chi$, which is initially heavy and lies at the origin. As $\varphi$ rolls down its classical potential it passes through a Symmetry Breaking Point (SBP). At that point the effective mass squared $\chi$ switches from a large positive to a negative value. This triggers an explosive production of $\chi$ particles. Due to their interactions, these particles create a very steep quantum potential for the quintessence, which halts the run of $\varphi$ almost instantaneously and traps it at SBP. We therefore often refer to the $\chi$ field as the "trapping field". The trapping mechanism is inspired by ref. [22]. However, instead of $\chi$ becoming massless, as suggested in that work, in our model we consider the mass of $\chi$ to be tachyonic at SBP.

An attractive feature of this scenario is that conclusions are insensitive to the curvature of the potential. Barring the arguments of naturalness, we don't need to impose any constraints on the flatness of the potential at low 
energies. Only the height of the potential at SBP is crucial. Therefore, quantum corrections do not have such a strong negative impact on this scenario as compared to the conventional quintessence models. Moreover, as the quintessence field can be trapped before it reaches the Planck scale, the effects of Planck-suppressed nonrenormalisable terms can also be neglected. Another benefit of this scenario is that the particle production during the trapping phase can be responsible for reheating the universe. As it is well known, reheating can be a challenging issue for generic quintessential inflation models [11]. Finally, at the minimum of the potential both fields, $\varphi$ and $\chi$, are heavy, avoiding the above mentioned 5 th force problems ${ }^{1}$

The fact that the trapping field has a negative mass squared at SBP brings some technical difficulties. To make the potential bounded from bellow, we must include a self-interaction term $\lambda \chi^{4}$. Such an interaction term makes the mode functions of the $\chi$ fields evolve non-linearly, which in turn affects the particle production and therefore the trapping (as well as reheating) efficiency. This is an important difference between the tachyonic trapping mechanism

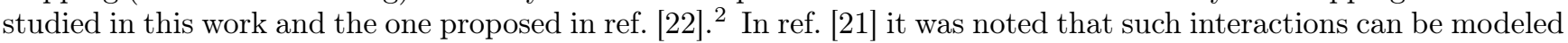
as an additional contribution to the effective mass of the trapping field. If that contribution is too large, the $\chi$ field becomes too heavy to be excited, reducing the efficiency of the trapping and the total energy density stored in $\chi$ particles.

In this work we study the effects of the non-linear evolution more carefully. We develop analytical methods for computing them and compare with the numerical simulations, showing a very good agreement. The former method makes it possible to scan a large space of parameter values and find regions where the trapping is efficient.

The paper is organised as follows: in section $[\Pi$ we introduce and motivate our model and discuss its basic features. The computation of non-linear effects is done in several steps. First, in section III we discuss the particle production ignoring the self-interaction term. The latter is accounted for by two different methods. If the suppression of particle production is small, we can take them into account perturbatively, as it is demonstrated in section IV] In the opposite regime, where non-linear effects are very strong, the resonantly produced particles cause "non-linear blocking", whereby newly created particles terminate any further production. This is discussed in section V In section VI we utilise the developed method to explore the space of parameter values that lead to efficient trapping and conclude in section VII. Finally in the Appendix we collect some mathematical formulas and more technical derivation steps.

In this work we use natural units such that $c=\hbar=1$ and the reduced Planck mass is $m_{\mathrm{pl}}=(8 \pi G)^{-1 / 2} \simeq$ $2.44 \times 10^{18} \mathrm{GeV}$.

\section{THE MODEL}

Consider a simple toy model, which contains two scalar fields: a real scalar field $\varphi$ and a complex scalar field $\Psi$. We write the Lagrangian of the model as

$$
\mathcal{L}=-\frac{1}{2} \partial_{\mu} \varphi \partial^{\mu} \varphi-\frac{1}{2} \partial_{\mu} \Psi \partial^{\mu} \Psi^{*}-V(\varphi,|\Psi|)
$$

where the potential $V(\varphi,|\Psi|)$ is of the form

$$
V(\varphi,|\Psi|)=V(\varphi)+\frac{1}{4} \lambda\left(\Psi \Psi^{*}-f^{2}\right)^{2}+\frac{1}{2} g^{2} \Psi \Psi^{*}\left(\varphi-\varphi_{\mathrm{SBP}}\right)^{2} .
$$

Generically, quintessential inflation models require the potential $V(\varphi)$ of the scalar field to feature two very flat plateaus at vastly different energy scales [11. These flatness conditions allow the potential energy of the field to dominate over the kinetic one, which is needed to drive the accelerated expansion of the universe. The plateau at high energies is supposed to provide a (quasi-)exponential expansion during inflation and the second plateau should be responsible for the accelerated expansion of the late universe. To accommodate for such a huge difference in energy scales at both epochs, the gradient of $V(\varphi)$ between the plateaus must be very large. Therefore, typically, after the end of inflation, the universe enters into the period of kination, where the energy budget is dominated by the kinetic energy of the scalar field [30, 31].

One concrete realisation of such a potential in the context of $\alpha$-attractors was suggested in ref. [21], where the mechanism of the tachyonic trap is also introduced. In the current work we are not interested in the detailed shape

\footnotetext{
${ }^{1}$ One could also consider adding a new challenge to quintessence (and therefore to quintessential inflation) scenarios from recent measurements of the Hubble constant $H_{0}$. The value of $H_{0}$, as determined from the CMB measurements [2], is lower than the one resulting from local determinations 23, 24, leading to the so called "Hubble tension" 25. Simple quintessence scenarios seem to exacerbate this tension according to ref. [26]. Therefore, if the tension is eventually confirmed, this will make simple quintessence scenarios with the equation of state $w>-1$ disfavoured. The scenario discussed in the current work does not suffer from such a problem because both fields are eventually trapped, which renders them non-dynamical.

2 A variation on the particle production mechanism studied in ref. 22] is also widely used to reheat the universe in quintessential inflation models [27-[29]. It is called the "instant reheating" mechanism.
} 
of $V(\varphi)$. The only assumptions we make about this part of the potential is that it contains a plateau at high energy scales to provide inflation and a very large gradient afterwards, in order to accommodate very small dark energy scale. In contrast to typical quintessential inflation scenarios, tachyonic trapping mechanism allows us to dispense with the second, low energy plateau. Indeed, the only requirement for $V(\varphi)$ at low energies is the height of the potential is $V_{\mathrm{SBP}} \equiv V\left(\varphi_{\mathrm{SBP}}\right) \simeq V_{\mathrm{vac}} \simeq 10^{-12} \mathrm{eV}^{-4}$, the exact shape being immaterial. This is a very important benefit of this scenario, as we don't need to worry about radiative corrections, which could otherwise spoil attractive quintessence models [27].

The second term in eq. (2) is the spontaneous symmetry breaking potential of $\Psi$, where $f$ is the symmetry breaking scale. And the last term in the potential specifies interactions between $\varphi$ and $\Psi$. To see the effects of such an interaction let us decompose $\Psi$ into its radial and angular components as $\Psi \equiv \chi \mathrm{e}^{\mathrm{i} \theta / m_{\mathrm{pl}}}$. We can thus clearly see that the $\chi$ field is heavy for $|\varphi| \gg\left|\varphi_{\mathrm{SBP}}\right|$ and therefore anchored at $\langle\chi\rangle=0$. The angular component $\theta$ is mainly neglected in this paper, apart from a few brief comments later.

As the $\varphi$ field runs towards $\varphi_{\mathrm{SBP}}$, its stabilising effect onto $\chi$ disappears and the $U$ (1) symmetry is spontaneously broken. Hence the name "Symmetry Breaking Point" (SBP). But before this happens the whole sequence of events take place, some of which is the main subject of the current work.

To simplify the discussion we will rescale the $\varphi$ field as

$$
\varphi \rightarrow \varphi-\varphi_{\mathrm{SBP}}
$$

without loosing generality. Therefore, neglecting the angular component $\theta$, we can write our working potential as

$$
V(\varphi, \chi)=V(\varphi)+\frac{1}{2} g^{2} \chi^{2} \varphi^{2}+\frac{1}{4} \lambda\left(\chi^{2}-f^{2}\right)^{2},
$$

which we use to model the processes close to $\varphi \simeq \varphi_{\mathrm{SBP}}=0$.

As discussed above, due to the large gradient of $V(\varphi)$, the universe is assumed to be dominated by the kinetic energy of the $\varphi$ field after inflation up until the first passage of SBP. We might consider this as an additional motivation for unifying inflation with dark energy models, as opposed to pure quintessence models, within the context of the tachyonic trapping mechanism: inflation provides the necessary conditions required for an effective trapping and reheating of the universe.

During kination the field is oblivious of its potential and the field's homogeneous component is governed by a simple equation of motion

$$
\ddot{\varphi}+3 H \dot{\varphi}=0,
$$

where $H$ is the Hubble parameter. In this regime $H$ is given by

$$
H \simeq \frac{|\dot{\varphi}|}{\sqrt{6} m_{\mathrm{pl}}} .
$$

It is easy to integrate eq. (5) and find

$$
\begin{aligned}
& \varphi=\sqrt{\frac{2}{3}} \operatorname{sign}(v) \ln \left(1+\sqrt{\frac{3}{2}} \frac{|v| t}{m_{\mathrm{pl}}}\right) m_{\mathrm{pl}}, \\
& \dot{\varphi}=v \mathrm{e}^{-\sqrt{\frac{3}{2} \frac{\varphi / m_{\mathrm{pl}}}{\operatorname{sign}(v)}}} .
\end{aligned}
$$

In these expressions time is defined such that $t_{\mathrm{SBP}}=0$ and

$$
v \equiv \dot{\varphi}_{\mathrm{SBP}}
$$

is the field velocity at SBP assuming no particle production. To simplify the notation, we will take $v>0$ in the rest of the paper.

Initially, for large $|\varphi|$ values, the trapping field is very heavy and anchored at the origin. The mode functions of $\chi$ satisfy the equation

$$
\ddot{\chi}_{k}+3 H \dot{\chi}_{k}+\omega_{k}^{2} \chi_{k}=0,
$$

where

$$
\omega_{k}^{2}=k^{2}+g^{2} \varphi^{2}-\lambda f^{2} .
$$


The initial conditions are give by the Bunch-Davies vacuum state, which is defined by the set of positive frequency modes:

$$
\chi_{k, \mathrm{vac}}=\frac{a^{-1}}{\sqrt{2 \omega_{k}}} \mathrm{e}^{-\mathrm{i} \int^{t} \omega_{k} \mathrm{~d} t^{\prime}} .
$$

Such an assumption is justified as for large $g^{2} \varphi^{2}$ values $\chi_{k}$ is heavy and cannot be excited. Interesting processes start when $\varphi$ approaches SBP.

As the field $\varphi$ runs close to $\varphi_{\mathrm{SBP}}=0$, the effective mass of the $\chi$ field vanishes, as can be seen from the definition of the potential in eq. (4). Moreover, inevitably for a range of values $\Delta \varphi_{\text {nad }}$ the rate at which that mass changes becomes non-adiabatic. This leads to the, so called, resonant production of $\chi$ particles as described in ref. [32]. Such particles create and effective linear potential for the $\varphi$ field, as we demonstrate later. If the production is strong enough, the linear potential is so steep that it halts the evolution of $\varphi$ and anchors it at $\varphi_{\text {SBP. A very }}$ similar process is described in detail in ref. [22]. In our model, there is an additional, and in some parameter space dominant, contribution to the particle production. As the potential has a tachyonic direction at SBP (see eq. (4)) one needs to account for the additional contribution to the particle production via the process called the tachyonic resonance [33.

To properly study the growth of such a linear potential we need to account for the effects of interaction terms in eq. (4). This is done by employing the Hartree approximation in our analytical computations as well as numerical simulations. Effectively this constitutes to replacing $\chi^{3} \rightarrow 3 \chi\left\langle\chi^{2}\right\rangle$. In particular, once such interactions are included, we need to update eq. (5) as

$$
\ddot{\varphi}+3 H \dot{\varphi}+g^{2}\left\langle\chi^{2}\right\rangle \varphi=0,
$$

where $\left\langle\chi^{2}\right\rangle$ is an expectation value computed as

$$
\left\langle\chi^{2}\right\rangle(t)=\frac{1}{2 \pi^{2}} \int_{0}^{\infty} k^{2}\left[\left|\chi_{k}(t)\right|^{2}-\frac{1}{2\left|\omega_{k}(t)\right|}\right] \mathrm{d} k,
$$

where the second term is included to subtract one loop contributions from $\chi$ particles 22 .

As one can clearly see from eq. (13), any $\chi$ particle production generates an effective potential for the $\varphi$ field. If the production is very efficient, the effective potential becomes so steep that it halts the run of $\varphi$ towards the minimum of $V(\varphi)$ and brings it back towards SBP, where it oscillates with an exponentially decaying amplitude.

The spontaneous symmetry breaking potential of $\Psi$ contain self-interaction terms. These terms make the equation of motion of the $\chi$ mode functions non-linear. Such non-linearities are the main subject of the study in this work.

To account for self-interactions in the evolution of $\chi_{k}$ we use the Hartree approximation too. In this case it corresponds to replacing the non-linear term by an additional (time dependent) contribution to the mass of the trapping field. That is, the full equation of motion for $\chi_{k}$ must be written as (c.f. eq. 10)

$$
\ddot{\chi}_{k}+3 H \dot{\chi}_{k}+\left[k^{2}+g^{2} \varphi^{2}-\lambda f^{2}+3 \lambda\left\langle\chi^{2}\right\rangle\right] \chi_{k}=0 .
$$

The last two terms make our scenario very different from the one discussed in ref. [22, where such terms are absent.

We use eqs. 13)- 15) together with the vacuum initial conditions in eq. 12 to study the full system numerically. In these simulations we integrate the large system of coupled differential equations 115 for a very broad range of $k$ values. But to make the analytical progress we utilise a number of approximations. Eventually the results of analytical computations are compared with the numerical simulations to confirm their accuracy.

\section{EVOLUTION NEGLECTING SELF-INTERACTIONS}

The first approximation that we can make is to neglect the expansion of the Universe. Indeed, the particle production is effective over a very small time interval $\Delta t$

$$
\frac{v \Delta t}{m_{\mathrm{pl}}} \ll 1
$$

Expanding eqs. (7) and (8) in terms of this small quantity and keeping only the highest order terms we find

$$
\begin{aligned}
& \varphi \simeq v t \ll m_{\mathrm{pl}}, \\
& \dot{\varphi} \simeq v=\mathrm{const},
\end{aligned}
$$


which are valid during the first passage of SBP.

On the other hand, applying the condition in eq. (16) to eq. (6) we find

$$
H_{\mathrm{SBP}} \Delta t \ll 1
$$

where

$$
H_{\mathrm{SBP}} \simeq \frac{v}{\sqrt{6} m_{\mathrm{pl}}} .
$$

Therefore, it is safe to neglect the Hubble expansion, i.e. the scale factor can be set to $a=1$, when analytically computing particle production during the first passage of $\mathrm{SBP} \mathrm{B}^{3}$

If the the particle production is sufficiently effective, each subsequent passage of SBP results in more $\chi$ particles being produced. Due to interactions, these particles backreact onto the motion of $\varphi$ and result in an exponential decrease of its oscillation amplitude. Therefore, the understanding of the first burst of particle production is important to be able to determine the efficiency of the trapping mechanism as a whole.

Self-interactions of the $\chi$ field can affect such an efficiency substantially in some of the parameter space. This can be seen from eq. 15, , where the $\left\langle\chi^{2}\right\rangle$ term can be interpreted as an additional, time dependent contribution to the effective mass of the $\chi$ field. As new particles are produced, $\left\langle\chi^{2}\right\rangle$ grows rapidly. But the growth of $\left\langle\chi^{2}\right\rangle$ also suppresses further particle production. In some cases, the growth of $\left\langle\chi^{2}\right\rangle$ can be so fast that it blocks any further particle production once it even barely started. We call this effect a "non-linear blocking".

However, to properly account for the non-linear effects onto the efficiency of particle production, and therefore the trapping, we first consider the case without self-interactions in this section. In the next section, we include non-linearities "perturbatively", if they are small, or compute the effects of non-linear blocking in section (V), if non-linearities are strong.

In the narrow window of particle production we apply the condition in eq. (16), which also leads to the conditions in eqs. (17) and 20). Therefore, without the non-linear term, one can write eq. 10) during the first passage of SBP as

$$
\ddot{\chi}_{k}^{(0)}+\omega_{k}^{2} \chi_{k}^{(0)} \simeq 0
$$

where $\omega_{k}^{2}$ is given by

$$
\omega_{k}^{2} \simeq k^{2}-\lambda f^{2}+g^{2} v^{2} t^{2} .
$$

Eq. (21) can be solved exactly in terms of Parabolyc Cylinder Functions (PCF) ${ }^{4}$ But to make generalisations and the connection to the existing literature easier, we use the WKB approximate expressions sufficiently far from

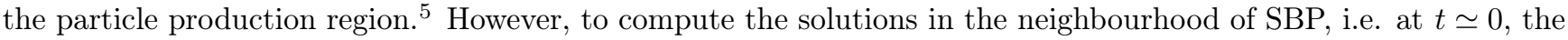
use of $\mathrm{PCF}$ is essential.

A similar computation is provided in ref. [32] where only the parametric particle production is considered. While the work in ref. 33] provides a similar computation but with parameters that make the tachyonic particle production dominant. In our case, both regimes are relevant. Therefore, we develop a computation which allows to account for both possibilities simultaneously.

Let us first denote the time $t_{ \pm}$such that

$$
t_{ \pm}= \begin{cases}0 & \text { for modes with } k^{2} \leq \lambda f^{2} \\ \omega_{k}^{2}\left(t_{ \pm}\right)=0 & \text { for modes with } k^{2}>\lambda f^{2}\end{cases}
$$

and sing $\left(t_{ \pm}\right)= \pm 1$. Then, in the region $t \ll t_{k-} \omega_{k}^{2}$ changes adiabatically, i.e. $\left|\dot{\omega}_{k}\right| \ll\left|\omega_{k}\right|^{2}$ and $\left|\ddot{\omega}_{k}\right| \ll\left|\omega_{k}\right|^{3}$, and we can write the WKB solution of eq. 21] as

$$
\chi_{k-}(t)=\frac{\alpha_{k-}}{\sqrt{2 \omega_{k}}} \mathrm{e}^{-\mathrm{i} \int_{t_{0}}^{t} \omega_{k} \mathrm{~d} t^{\prime}}+\frac{\beta_{k-}}{\sqrt{2 \omega_{k}}} \mathrm{e}^{\mathrm{i} \int_{t_{0}}^{t} \omega_{k} \mathrm{~d} t^{\prime}}
$$

where $\chi_{k-}(t) \equiv \chi_{k}\left(|t| \gg t_{k-}\right)$.

Long after the first burst of particle production, at $t \gg t_{k+}$, the rate of change of $\omega_{k}$ is again adiabatic and we can write

$$
\chi_{k+}(t)=\frac{\alpha_{k+}}{\sqrt{2 \omega_{k}}} \mathrm{e}^{-\mathrm{i} \int_{t_{+}}^{t} \omega_{k} \mathrm{~d} t^{\prime}}+\frac{\beta_{k+}}{\sqrt{2 \omega_{k}}} \mathrm{e}^{\mathrm{i} \int_{t_{+}}^{t} \omega_{k} \mathrm{~d} t^{\prime}}
$$

\footnotetext{
3 When solving the equations numerically we do not make this approximation.

${ }^{4}$ We summarise a few relevant properties of PCF and derive other useful relations in the appendix, section A

5 This region has to be close enough for the condition in eq. 16 to be satisfied. However, as we will see later, this requirement is not restrictive at all.
} 
where $\chi_{k+}(t) \equiv \chi_{k}\left(t \gg t_{k+}\right)$. In both cases Bogoliubov coefficients are normalised as $\left|\alpha_{k \pm}\right|^{2}-\left|\beta_{k \pm}\right|^{2}=1$.

As was mentioned above, initially the trapping field is heavy and remains in its vacuum state. This corresponds to choosing the positive frequency mode of eq. (24), i.e. $\alpha_{k-}=1$ and $\beta_{k-}=0$.

To find the connection formulas between coefficients $\alpha_{k-}, \beta_{k-}$ and $\alpha_{k+}, \beta_{k+}$ one can use standard methods employed in quantum mechanics (this is discussed in many textbooks on quantum mechanics, for example [34]).

For modes with the wavenumber $k^{2}>\frac{2 g v}{3^{3 / 2}}+\lambda f^{2}, \omega_{k}^{2}$ is always positive and remains adiabatic. Therefore, such modes do not undergo any amplification and WKB solutions in eqs. (24) and (25) can be "connected directly", i.e. $\alpha_{k-}=\alpha_{k+}$ and $\beta_{k-}=\beta_{k+}$. For modes with smaller $k$ value the two adiabatic regimes are interrupted by a non-adiabatic regime, in the case of $\lambda f^{2}<k^{2}<\frac{2 g v}{3^{3 / 2}}+\lambda f^{2}$, or also by a regime with a negative $\omega_{k}^{2}<0$, in the case of $k^{2}<\lambda f^{2}$. In those cases the connection between $\alpha_{k \pm}$ and $\beta_{k \pm}$ coefficients is more complicated, which is the manifestation of the particle production.

When non-adiabaticity is broken, the two adiabatic regions can be connected using the solutions in terms of PCF

$$
\chi_{k}=a_{k} W(\kappa, \tau)+b_{k} W(\kappa,-\tau)
$$

where we defined

$$
\begin{aligned}
\kappa & \equiv \frac{\lambda f^{2}-k^{2}}{2 g v}, \\
\tau & \equiv \sqrt{2 g v} t
\end{aligned}
$$

Generically such solutions, in terms of PCF constitute a very good approximation. But in our case they are exact due to the form of $\omega_{k}$ in eq. 22, This being the case, we use the solution in eq. 26) in the whole region where $\omega_{k}^{2}$ is negative to simplify the derivation. Although, we could also make use of the WKB approximation in the regime where $\omega_{k}^{2} \ll 0$, similarly to what we do in section $\mathrm{V}$.

To derive the connection formulas, on can be extended eq. (26) to regions $|t| \gtrsim\left|t_{ \pm}\right|$, where the WKB expressions as well as the expression in eq. (26) are both sufficiently good approximations $\sqrt[6]{6}$ We first match the WKB expression in eq. (24) with the one in eq. (26). This procedure gives (see eqs. A16) and (A17)

$$
\begin{aligned}
& a_{k}=\mathrm{i}\left(\frac{\sqrt{1+\mathrm{e}^{2 \pi \kappa}}-\mathrm{e}^{\pi \kappa}}{2 \sqrt{2 g v}}\right)^{1 / 2} \mathrm{e}^{-\mathrm{i}\left(\theta_{k}+\varphi_{k}\right)}, \\
& b_{k}=\left(\frac{\sqrt{1+\mathrm{e}^{2 \pi \kappa}}+\mathrm{e}^{\pi \kappa}}{2 \sqrt{2 g v}}\right)^{1 / 2} \mathrm{e}^{-\mathrm{i}\left(\theta_{k}+\varphi_{k}\right)},
\end{aligned}
$$

where we used vacuum initial conditions at $t_{0} . \theta_{k}$ in the above expressions is the phase accumulated from the initial moment $t_{0}$ to $t_{-}$

$$
\theta_{k} \equiv \int_{t_{0}}^{t_{-}} \omega_{k} \mathrm{~d} t
$$

and $\varphi_{k}$ is defined in eq. A20 as

$$
2 \varphi_{k} \equiv \frac{1}{2} \pi+\arg \left[\Gamma\left(\frac{1}{2}+\mathrm{i} \kappa\right)\right]+\kappa(1-\ln |\kappa|)
$$

To find the final value of $\chi_{k}$, after the resonance is over, we do the second matching of eq. 26) to the WKB solution in the $t>t_{+}$region in eq. 25. This gives

$$
\begin{aligned}
& \alpha_{k+}=\sqrt{1+\mathrm{e}^{2 \pi \kappa}} \mathrm{e}^{-\mathrm{i} \varphi_{k}} \\
& \beta_{k+}=\mathrm{e}^{\pi \kappa} \mathrm{e}^{-2 \mathrm{i}\left(\theta_{k}+\frac{\pi}{4}\right)}
\end{aligned}
$$

In summary, after the particle production is over, the mode functions of the trapping field evolve according to eq. 25 with the constants given in eqs. (33) and 34 .

\footnotetext{
${ }^{6}$ Note, that such a region does not exist in general. But it certainly does in our case.
} 
We can use this result to compute the occupation number $n_{k}$ defined as 32

$$
n_{k}=\frac{\left|\omega_{k}\right|}{2}\left[\frac{\left|\dot{\chi}_{k}\right|^{2}}{\left|\omega_{k}\right|^{2}}+\left|\chi_{k}\right|^{2}\right]-\frac{1}{2}
$$

which is approximately constant in the WKB region with $\omega_{k}^{2}>0$. Plugging in eq. 25 into eq. (35) with $\alpha_{k+}$ and $\beta_{k+}$ provided in eqs. (33) and 34 we find

$$
n_{k}^{(0)}=\left|\beta_{k+1}\right|^{2}=\mathrm{e}^{\pi \frac{\lambda f^{2}-k^{2}}{g v}},
$$

where the superscript ' $(0)$ ' indicates that this quantity is computed neglecting non-linearities.

Integrating this expression according to

$$
n_{\chi}=\frac{1}{2 \pi^{2}} \int_{0}^{\infty} k^{2} n_{k} \mathrm{~d} k
$$

gives the particle number density. At the "zeroth order" in non-linearities this expression leads to

$$
n_{\chi}^{(0)}=\left(\frac{\sqrt{g v}}{2 \pi}\right)^{3} \mathrm{e}^{\pi \frac{\lambda f^{2}}{g v}} .
$$

\section{THE EFFECT OF SELF-INTERACTIONS}

In the previous section we computed the particle number density after the first passage of SBP neglecting selfinteractions of the trapping field $\chi$. Such interactions introduce non-linear terms in the equation of motion of $\chi_{k}$, which makes it impossible to find exact analytical solutions. Unfortunately, in a large parameter space selfinteractions affect the final particle number density considerably and cannot be neglected. In this and the next sections we develop methods to compute the effects of such non-linearities.

First, we are going to employ the Hartree approximation as was already mentioned in the discussion leading to eq. (15). Such an approximation should be sufficient when the non-linear term is small. In the opposite regime, when it becomes large, the Hartree approximation breaks. However, it is reasonable to think that this does not affect the final results much. The reason being that large non-linear term blocks any further particle production. Therefore, we only need to find the evolution of $\left\langle\chi^{2}\right\rangle$ term until just before the non-linear blocking, where we can use the results of section III.

In the current section we are going to study the parameter region where the effects of non-linear evolution can be accounted for perturbatively. The case of strong non-linearities will be considered in the next section separately.

Our method consists in solving for $\chi_{k}$ iteratively. At the zeroth order we take the solution derived in the previous section, which provides us with the method to compute $\left\langle\chi^{2}\right\rangle^{(0)}(t)$. At the next order we solve the equation (c.f. eqs. (21) and (22)

$$
\ddot{\chi}_{k}^{(1)}+\left(\omega_{k}^{2}+\delta m^{2}\right) \chi_{k}^{(1)}=0
$$

Notice, that at this order we included an additional contribution to the effective mass squared

$$
\delta m^{2} \equiv 3 \lambda\left\langle\chi^{2}\right\rangle_{0}^{(0)}=\text { constant }
$$

where $\left\langle\chi^{2}\right\rangle_{0}^{(0)} \equiv\left\langle\chi^{2}\right\rangle^{(0)}(t=0)$ is the expectation value of $\chi^{2}$ computed at zeroth order and evaluated at the time $t=0$, i.e. at SBP.

For modes that satisfy $\lambda f^{2}<k^{2}<\frac{2 g v}{3^{3 / 2}}+\lambda f^{2}$ we cannot use the WKB solutions in the neighbourhood of $t=0$ as $\omega_{k}^{2}$ does not evolve adiabatically in that region. Thus we will use the exact solutions in terms of PCF in eq. (26). For modes with $k^{2} \ll \lambda f^{2}$ WKB approximation does give a good solution at $t \simeq 0$. However, to simplify the argument and provide a unified framework we will also use the solution in eq. (26).

Plugging eqs. (29) and (30) into eqs. 26) and (14) we find

$$
\left\langle\chi^{2}\right\rangle_{0}^{(0)}=\frac{1}{2 \pi^{2} \sqrt{2 g v}} \int_{0}^{\infty} k^{2}\left[W^{2}(\kappa, 0) \sqrt{1+\mathrm{e}^{2 \pi \kappa}}-\frac{1}{2 \sqrt{\kappa}}\right] \mathrm{d} k .
$$


The above integrand peaks at some $k_{*}$ value. For the most of the parameter space this value is such that $\left|\kappa_{*}\right|>1 / 2$, where $\kappa(k)$ is defined in eq. (27). This fact justifies the usage of an approximate value of $W(\kappa, 0)$ in eq. A6

$$
W^{2}(\kappa, 0) \simeq \frac{1}{2 \sqrt{|\kappa|}}
$$

Plugging it into eq. 41, we obtain

$$
\left\langle\chi^{2}\right\rangle^{(0)}(t=0) \simeq \frac{1}{4 \pi^{2}} \int_{0}^{\infty} k^{2} \frac{\sqrt{1+\mathrm{e}^{2 \pi \kappa}}-1}{\sqrt{\left|k^{2}-\lambda f^{2}\right|}} \mathrm{d} k .
$$

The integral can be computed using the Laplace's approximation. As the computation involves a few steps we summarise them in Appendix B. The final result depends on the ratio

$$
Q \equiv \pi \frac{\lambda f^{2}}{g v}
$$

For the $Q$ values in the range $0<Q<$ a few, the largest contribution to the integral comes from the mode (see eq. (B5)

$$
k_{* p}^{2} \simeq \frac{g v}{\pi} .
$$

In this case the approximate value of eq. 43 can be computed to be

$$
\left\langle\chi^{2}\right\rangle_{0 p}^{(0)} \simeq \frac{g v}{(2 \pi)^{3}} \sqrt{\frac{\pi / 2}{|1-Q|}} \mathrm{e}^{Q-1} .
$$

In the opposite regime, with very large $Q$, the biggest contribution to the integral comes from the modes (see eq. (B7)

$$
k_{* t}^{2} \simeq \frac{2 g v}{\pi} .
$$

Thus this is the regime were particle production is overwhelmingly dominated by the tachyonic particle production $\left(\lambda f^{2}=Q k_{* t}^{2} / 2 \gg k_{* t}^{2}\right)$. The expectation value of $\chi^{2}$ at $t=0$ in this case is

$$
\left\langle\chi^{2}\right\rangle_{0 t}^{(0)} \simeq \frac{g v}{2 \pi^{3}} \sqrt{\frac{\pi / 2}{\left|1-\frac{1}{2} Q\right|}} \mathrm{e}^{\frac{1}{2} Q-1}
$$

where we used eq. (B8).

The $\delta m^{2} \equiv 3 \lambda\left\langle\chi^{2}\right\rangle_{0}^{(0)}$ term only adds a positive constant contribution to $\omega_{k}^{2}$. Therefore, it is easy to deduce that at the 1 st order in this approximation the particle number density can be written as

$$
n_{\chi}^{(1)}=n_{\chi}^{(0)} \mathrm{e}^{-\pi \frac{3 \lambda\left\langle\chi^{2}\right\rangle_{0 i}^{(0)}}{g v}}
$$

where $\left\langle\chi^{2}\right\rangle_{0 i}^{(0)}$ is either $\left\langle\chi^{2}\right\rangle_{0 p}^{(0)}$ (for $Q<$ a few) or $\left\langle\chi^{2}\right\rangle_{0 t}^{(0)}$ (for $\left.Q \gg 1\right)$.

We compare this result with the numerical simulations in figure 1. The result in eq. 49 is shown as the black curves in the first column of plots. The blue dot-dashed line in that figure corresponds to $Q=1$. To the left of that line parametric particle production dominates. While on the right hand side, the tachyonic particle production dominates.

On the left hand side of the dot-dashed red line in figure 1 the self-interaction induced suppression factor is small, and we can apply the perturbative result in eq. 49 . However, as $\left\langle\chi^{2}\right\rangle_{0}^{(0)}$ becomes very large, this result is rendered inadequate. We draw the boundary between the two regions (which is shown by the red line) at $n_{\chi}^{(1)} / n_{\chi}^{(0)}=1 / 2$. This is equivalent to saying that the perturbative computation is used in the region that satisfies the condition

$$
\sqrt{Q-1} \mathrm{e}^{1-Q}<\frac{3 \lambda}{\ln 16(2 \pi)^{3 / 2}} .
$$

In the opposite regime, the non-linear blocking terminates particle production. We discuss this case in the next section. 


\section{THE STRONGLY NON-LINEAR REGIME}

The tachyonic mass of the $\chi$ field at SBP makes the particle production much more effective. A priori one would expect that this makes the trapping more efficient. However, to make the potential bounded from bellow, we need to introduce a self-coupling term. As we saw in the previous paragraph, this term suppresses the particle production. If the latter is very efficient, the non-linear blocking shuts it down completely.

The non-linear blocking happens due to the rapidly increasing $3 \lambda\left\langle\chi^{2}\right\rangle$ term. Once this term reaches $3 \lambda\left\langle\chi^{2}\right\rangle \simeq \omega_{k}^{2}$, the trapping field becomes too heavy for further excitations. We determine the exact proportionality constant from our numerical simulations. Indeed, we find that particle production is shut off at the moment $t_{\mathrm{nl}}$, when the condition

$$
\omega_{k_{* t}}^{2}\left(t_{\mathrm{nl}}\right)=-c \cdot 3 \lambda\left\langle\chi^{2}\right\rangle^{(0)}\left(t_{\mathrm{nl}}\right)
$$

is satisfied, where $c \simeq 2.15$.

In principle we can use eqs. 26, 29 and 30 to find the time evolution of $\left\langle\chi^{2}\right\rangle^{(0)}(t)$, but this is not very illuminating. Instead, we are going to use the WKB approximate relations. This is made possible by the fact that non-linear blocking happens only in the regime of strong tachyonic instability. This regime corresponds to very large $\kappa$ (defined in eq. (27)), where PCF $W(\kappa, \tau)$ can be approximated by their WKB expressions as in eq. A26).

Let us write the latter as

$$
\chi_{k} \simeq \frac{\alpha_{k}}{\sqrt{2\left|\omega_{k}\right|}} \mathrm{e}^{-\int_{0}^{t}\left|\omega_{k}\right| \mathrm{d} t}+\frac{\beta_{k}}{\sqrt{2\left|\omega_{k}\right|}} \mathrm{e}^{\mathrm{e}_{0}^{t}\left|\omega_{a}\right| \mathrm{d} t}
$$

where the constants $\alpha_{k}, \beta_{k}$ are normalised as $\alpha_{k} \beta_{k}^{*}-\alpha_{k}^{*} \beta_{k}=\mathrm{i}$. They can be related to $a_{k}, b_{k}$ in eq. A27) as

$$
\begin{aligned}
\alpha_{k} & =(8 g v \kappa)^{1 / 4} W(\kappa, 0) a_{k} \\
\beta_{k} & =(8 g v \kappa)^{1 / 4} W(\kappa, 0) b_{k}
\end{aligned}
$$

Plugging this expression into eq. (14) we find

$$
\left\langle\chi^{2}\right\rangle^{(0)}(t)=\frac{1}{4 \pi^{2}} \int_{0}^{\infty} \frac{k^{2}}{\left|\omega_{k}\right|}\left(\left|\alpha_{k}\right|^{2} \mathrm{e}^{-2 \int_{0}^{t}\left|\omega_{k}\right| \mathrm{d} t}+\left|\beta_{k}\right|^{2} \mathrm{e}^{2 \int_{0}^{t}\left|\omega_{k}\right| \mathrm{d} t}-1\right) \mathrm{d} k
$$

where we used the large $\kappa$ approximation of $W(\kappa, 0)$ (eq. (A6)). Evaluating this integral at $t=0$ eq. (48) is recovered. As the non-linear blocking only happens in the regime where tachyonic particle production is dominant, we concentrate on the $k_{* t} \ll \sqrt{\lambda} f$ mode, which is defined in eq. (47).

For sufficiently large $|t|$, the exponentially increasing term dominates eq. (55). Moreover, due to the smallness of $k_{* t}$, we can approximate $\omega_{k_{*}} \simeq \omega_{k=0}$ and write the expression in eq. 55) as

$$
\left\langle\chi^{2}\right\rangle^{(0)}(t) \simeq\left\langle\chi^{2}\right\rangle_{0 t}^{(0)} \mathrm{e}^{2 X(t)}
$$

$X(t)$ in the above is the integral given by

$$
X(t) \equiv \int_{0}^{t}\left|\omega_{k=0}\right| \mathrm{d} t=\frac{\varphi}{2 v}\left|\omega_{k=0}(\varphi)\right|+\frac{\lambda f^{2}}{2 g v} \arcsin \frac{g \varphi}{\sqrt{\lambda f^{2}}} .
$$

Finally to find the value of $t_{\mathrm{nl}}$ when the non-linear blocking happens we can plug eq. (56) into 51). Unfortunately, this leads to the transcendental expression

$$
\lambda f^{2}-g^{2} \varphi^{2}\left(t_{\mathrm{nl}}\right)=3 c \lambda\left\langle\chi^{2}\right\rangle_{0 t}^{(0)} \mathrm{e}^{2 X\left(t_{\mathrm{nl}}\right)}
$$

which cannot be solved analytically. It is valid in the regime $\lambda f^{2}>g^{2} \varphi^{2}$, so one would be tempted to expand it in terms of $g \varphi / \sqrt{\lambda} f$ and keep only a few lower order terms. We found, however, that this procedure gives a poor agreement with the numerical simulations. Therefore, in what follows we use the full expression and solve eq. (58) numerically.

The final particle number density, at large $\varphi$ values, can be computed using the equation 32 .

$$
\left\langle\chi^{2}\right\rangle(t) \simeq \frac{1}{2 \pi^{2}} \int_{0}^{\infty} k^{2} \frac{n_{k}(t)}{\left|\omega_{k}(t)\right|} \mathrm{d} k .
$$



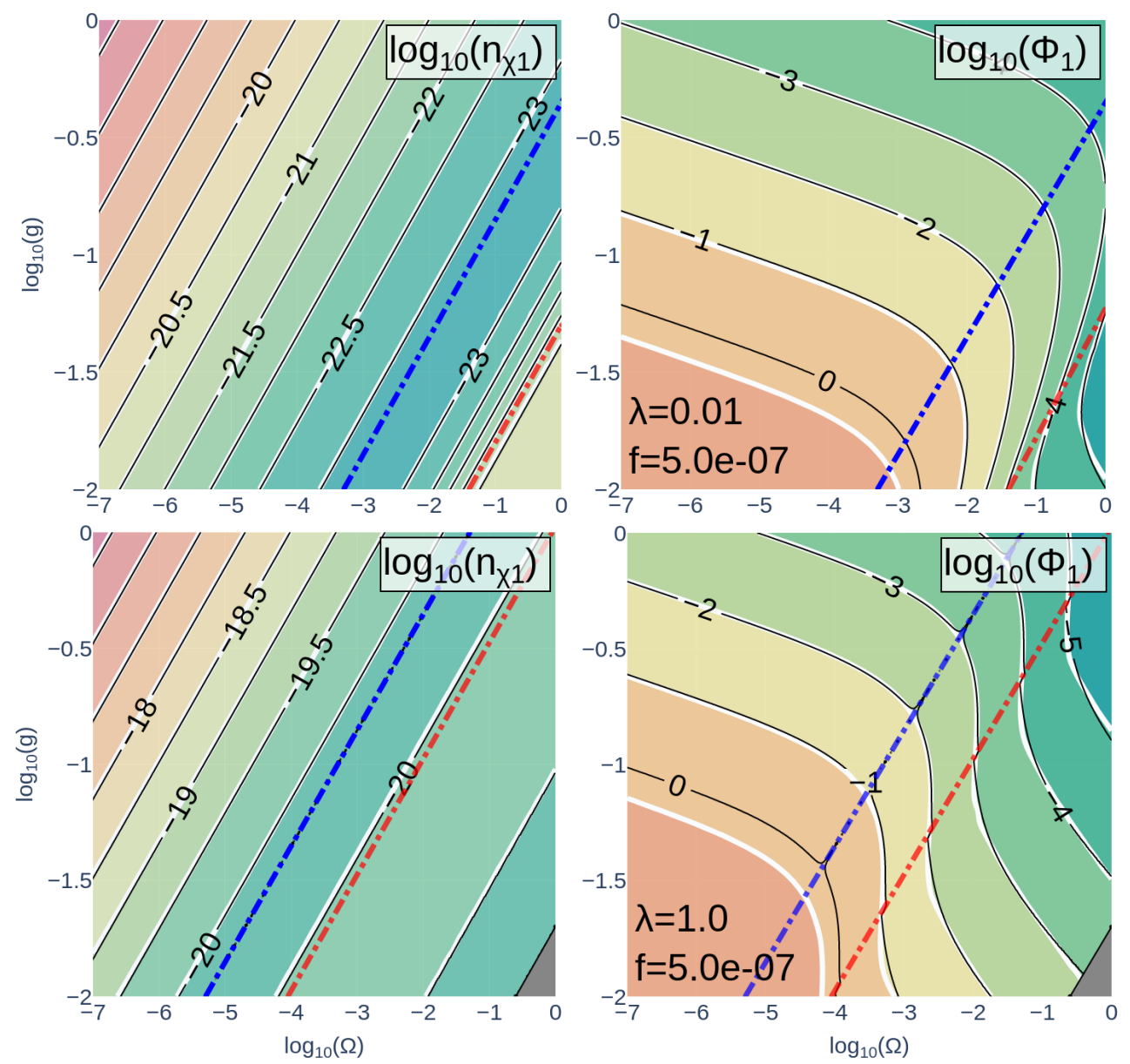

Figure 1. The comparison of the analytic computations (solid black curves) with the numerical simulations (white curves and colour bands). The upper row corresponds to $\lambda=0.01$ and the lower one to $\lambda=1$. We took $f=5 \times 10^{-7}$ in both of these models. On the left column we display the particle number density $n_{\chi}$ as the function of the interaction strength $g$ and $\Omega \propto v^{-2}$ (see eq. (71)). On the right column we show the amplitude of the first oscillation $\Phi_{1}$ in units of $m_{\mathrm{pl}}$. The blue dotdashed line corresponds to $Q=1$ (see eq. (44)) and separates the region where the parametric particle production dominates (left) from the one where particles are primarily produced by the tachyonic amplification (right). In the neighbourhood of the blue line the approximation used in eq. 42 is inadequate. This leads to some artefacts in the plots with large $\lambda$ values. On the right of the red dot-dashed line the condition in eq. 50 is violated, that is, the particle production is terminated by the non-linear blocking. As one can see from these plots, the agreement between numerical and analytic computation is very good. At $\Phi_{1}=m_{\mathrm{pl}}$ the expansion of the universe starts to be important as can be evidenced from the mismatch between black and white curves on the right column. The grey triangle in the bottom-right corner masks the region where our numerical simulations can no longer solve the equations reliably.

Substituting again $\omega_{k_{*}}^{2}(t) \simeq \omega_{k=0}^{2}(t)$ in the above expression we can factor out $\omega_{k}$, and write

$$
n_{\chi}=n_{\chi}\left(t_{\mathrm{nl}}\right) \simeq\left|\omega_{k=0}\left(t_{\mathrm{nl}}\right)\right|\left\langle\chi^{2}\right\rangle\left(t_{\mathrm{nl}}\right) .
$$

This analytic estimate gives a surprisingly good fit to numerical simulations as can be seen in figure 1. The method developed in this section is applied to computed the right hand side from the red, dot-dashed line in that figure, which corresponds to the regime where the condition in eq. (50) is broken.

\section{THE EFFICIENCY OF TRAPPING}

As discussed in section [II, the interaction between $\varphi$ and $\chi$ fields do not only affect the evolution of the $\chi$ field but it works the other way round too. The newly created $\chi$ particles backreact onto the motion of the $\varphi$ field as is demonstrated in eq. (13). It is clear from that expression that one can consider such a backreaction as a quantum mechanically generated effective potential. 
Sufficiently late after the first burst of particle production one can write $\omega_{k_{*}}^{2} \simeq g^{2} \varphi^{2}$. Plugging this approximation into eq. (59) and neglecting the rapidly oscillating terms (see ref. [32] for details) we obtain

$$
g^{2}\left\langle\chi^{2}\right\rangle(t) \simeq \frac{g n_{\chi}}{|\varphi|}
$$

where $n_{\chi}$ is given either in eq. (49), for weak non-linearities, or in eq. 60, if particle production is terminated by the non-linear blocking.

If the particle production is efficient enough and the backreaction is strong, we can make sure that the $\varphi$ field never reaches super-Planckian values. The need for super-Planckian $\varphi$ values in quintessential inflation models is often recognised as being problematic, as it makes difficult to justify the absence of non-renormalisable terms in the original action of the field [17. One can avoid this problem if the trapping is very strong.

Let us denote the oscillation amplitude of the $\varphi$ field as $\Phi$, and the amplitude after the first burst of particle production as $\Phi_{1}$. After every other passage of SBP the amplitude decreases. Therefore it is enough to impose the condition on the oscillation amplitude after the first passage of SBP

$$
\Phi_{1}<m_{\mathrm{pl}} \text {. }
$$

As the Universe expands, newly produced particles are diluted, therefore reducing the efficiency of the trapping [35]. To prevent this, we require that the time between each burst of particle production is less than the Hubble time. This condition is much stronger than the one in eq. (19). The latter only applies to the interval of the particle production. Now, we impose a similar condition to the duration of one oscillation. It is possible to show that such a condition is equivalent to the one eq. 62 .

If the expansion of the universe is neglected, we can write the equation of motion 13 as

$$
\ddot{\varphi}+g n_{\chi} \frac{\varphi}{|\varphi|} \simeq 0
$$

where we also used eq. [61]. It is easy to solve this equation (see ref. [22]). After passing $\mathrm{SBP} \varphi$ continues to increase until its initial kinetic energy density is transferred to the $\chi$ particles. This happens at a time

$$
t_{1}=\frac{v}{g n_{\chi 1}} .
$$

At that moment the $\varphi$ field amplitude is

$$
\Phi_{1}=\frac{1}{2} \frac{v^{2}}{g n_{\chi 1}} .
$$

Instead of rolling to the minimum of $V(\varphi)$, as the classical dynamics would dictate, the $\varphi$ field turns around and runs back towards SBP. At the SBP, the $\chi$ field is approximately massless again, and $\dot{\varphi} \simeq v$.

Plugging eq. 64 into 65 we find

$$
\Phi_{1}=\frac{1}{2} v t_{1}
$$

At the first passage of SBP the universe is dominated by the kinetic energy of the $\varphi$ field,

$$
\rho_{\text {kin }} \equiv \frac{1}{2} v^{2}
$$

and the Hubble parameter is given in eq. (19). Plugging this result into eq. (66) and using the bound in eq. 62 we find

$$
H_{\mathrm{SBP}} t_{1}<\sqrt{\frac{2}{3}} .
$$

As one can see, the requirement for sub-planckian field values also guarantees that the expansion of the universe can be neglected when computing the particle production during the trapping phase. We confirmed this using our numerical simulations too, which do include the Hubble expansion consistently.

One of the goals of this work is to find the parameter range where the trapping mechanism is efficient, that is, where the condition in eq. (62), or equivalently in eq. (68), is satisfied. To do that we use eq. (65) to scan over the large space of parameter values, with $n_{\chi 1}$ given either by the expression in eq. 49 or (60) depending on the value of $Q$.

The scanning is performed over the space of four independent parameters: $g, \lambda, f$ and $v$. However, the final constraints should not be too sensitive to the specifics of the model. The range of the $\varphi$ field values is quite narrow 
in the window where $\chi$ particles are produced, and the expression in eq. 177 should be a good approximation for a large range of models. For this reason we express the parameter ranges in figure 2 in terms of a physically more relevant quantity: the ratio of the potential to the kinetic energy at SBP

$$
\left.\Omega \equiv \frac{\rho_{\text {pot }}}{\rho_{\text {kin }}}\right|_{\text {SBP }} .
$$

The kinetic energy density is defined in eq. (67). In our particular model, the potential energy at SBP can be deduced from eq. (4)

$$
\left.\rho_{\text {pot }}\right|_{\text {SBP }}=V_{\text {vac }}+\frac{1}{4} \lambda f^{4},
$$

where $V_{\mathrm{vac}} \simeq 10^{-120} m_{\mathrm{pl}}^{4} \ll \lambda f^{4}$ is the vacuum energy density. Plugging in eq. 67) we can therefore write

$$
\Omega \simeq \frac{\lambda f^{4}}{2 v^{2}} .
$$

Let us now consider the possible range of parameter values that we should scan over. The lowest possible bound on $\Omega$ can be found by noting that $\rho_{\text {kin }}<V_{*}<m_{\mathrm{pl}}^{4}$. To avoid the second period of inflation at SBP we also impose the upper bound $\Omega<1$. Putting these two bounds together one can write

$$
\frac{\lambda f^{4}}{4 m_{\mathrm{pl}}^{4}}<\Omega<1 .
$$

The maximum value of $f$ is constrained from observations. As the symmetry at SBP is broken one forms a network of cosmic strings. The tension of such strings is proportional to the symmetry breaking scale $G \mu=f^{2} / m_{\mathrm{pl}}^{2}$, where $G$ is Newton's constant. The tightest constraints on $G \mu$ come from CMB measurements, which give $G \mu<10^{-7}$ [36 38] (see however ref. [39]). In principle $f$ is also bounded from bellow by the requirement that the symmetry breaking scale is larger than the scale of the big bang nucleosynthesis (BBN). However, such a bound is much weaker than the requirement for effective trapping.

The lowest value of $g$ is dictated by the gravitationally induced interactions. While the upper bounds on $g$ and $\lambda$ are dictated by the requirement of perturbativity. We will take that value to be 1 .

Finally, we must consider that before SBP the $\chi$ field must evolve adiabatically. That is

$$
\left|\frac{\dot{\omega}_{k=0}}{\omega_{k=0}^{2}}\right|_{\varphi_{\mathrm{ini}}} \ll 1
$$

where $\varphi_{\text {ini }}$ is the field value at the end of inflation and 7

$$
\omega_{k=0}^{2} \simeq g^{2} \varphi^{2}-\lambda f^{2} .
$$

Between $\varphi_{\mathrm{ini}}$ and SBP the universe is dominated by the kinetic energy of the $\varphi$ field. Therefore, plugging in eq. (8) into (74) we find

$$
\left|\frac{\dot{\omega}_{k=0}}{\omega_{k=0}^{2}}\right|=\frac{v}{g} \frac{|\varphi|}{\left(\varphi^{2}-\frac{\lambda f^{2}}{g^{2}}\right)^{3 / 2}} \mathrm{e}^{-\sqrt{\frac{3}{2}} \frac{\varphi}{m_{\mathrm{p} 1}}},
$$

where $g^{2} \varphi^{2} \gg \lambda f^{2}$. This function has a minimum $\left|\dot{\omega}_{k=0} / \omega_{k=0}^{2}\right| \simeq \frac{v}{g} \frac{3}{8} \mathrm{e}^{2} / m_{\mathrm{pl}}^{2}$ at $\varphi \simeq-2 \sqrt{\frac{2}{3}} m_{\mathrm{pl}}^{2}$. Imposing the bound in eq. 73 we find

$$
\Omega \gg \frac{\lambda f^{4}}{g^{2} m_{\mathrm{pl}}^{4}} .
$$

The above bound is somewhat stronger than the one in eq. $(72)$, however we find that it is still much weaker than the bound in eq. (62).

The results of the parameter space scanning are provided in figure 2 In each plot of that figure we draw a curve for a given value of $\lambda$ and $f$ that corresponds to $\Phi_{1}=m_{\mathrm{pl}}$ as computed using eq. (65). In the space above a given

7 This expression neglects the expansion of the universe. However, we checked that the results do not change substantially if we include it. 

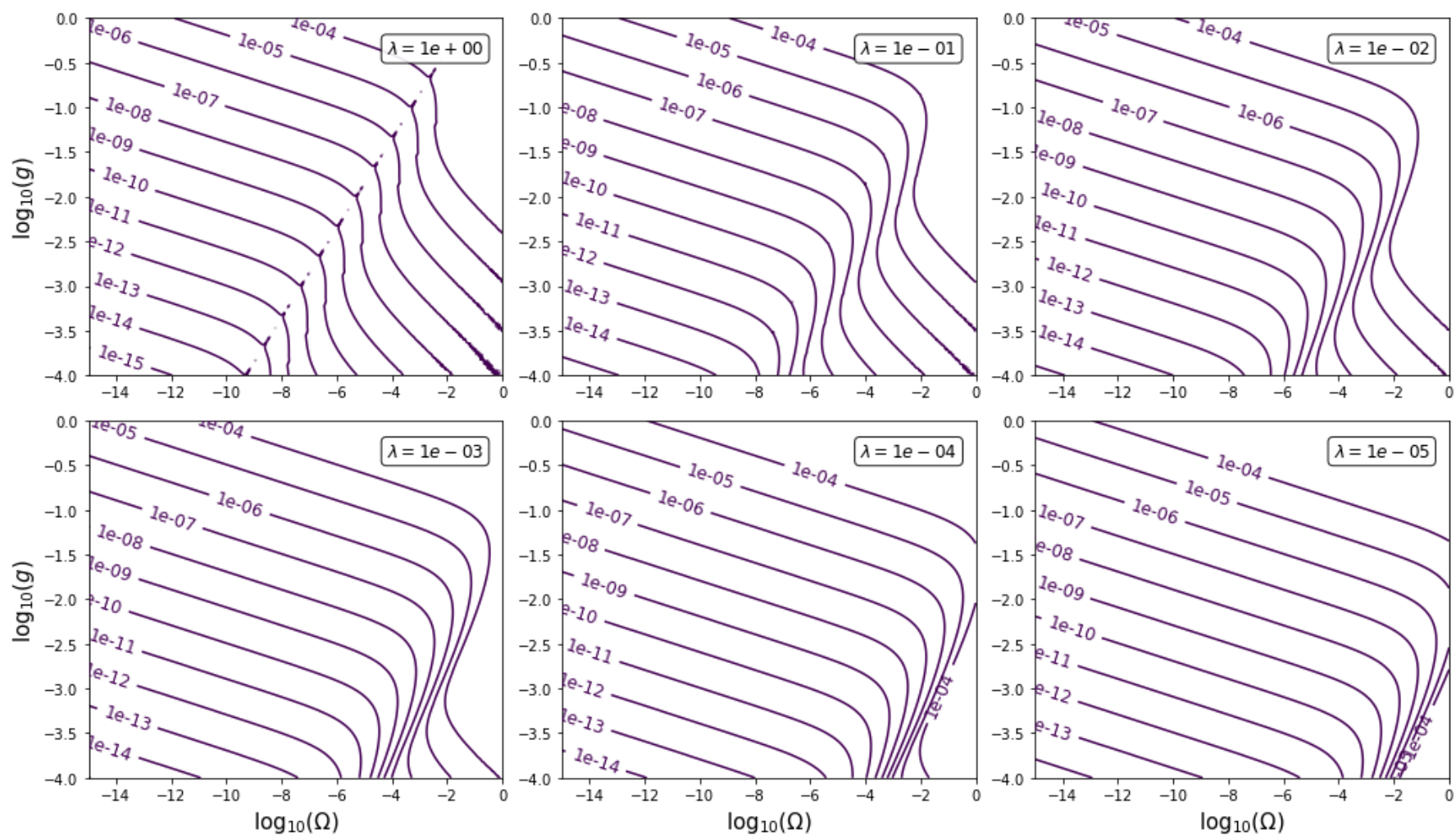

Figure 2. The 4-dimensional parameter space $\lambda, f, g$ and $v$ for which the trapping mechanism is effective. Each curve corresponds to a given value of $f$ (shown in the labels) that results in $\Phi_{1}=m_{\mathrm{pl}}$ (neglecting the expansion of the universe). Above and to the right of a that curve $\Phi_{1}<m_{\mathrm{pl}}$ for the fixed values of $\lambda$ and $f$. That is, increasing $g$ and/or decreasing $v$, enhances the efficiency of the trap. The definition of the horizontal axis is shown in eq. (69).

curve, which corresponds to a fixed $\lambda$ and $f$ but larger values of $g$ and/or smaller values of $v$, the trapping is more efficient and results in a smaller oscillation amplitude $\Phi_{1}<m_{\mathrm{pl}}$.

The main goal of the paper is to investigate how non-linearities affect the oscillation amplitude in the trap. A more detailed study of subsequent processes falls outside the scope of this work. For completeness, we only summarise the main points discussed in ref. [21].

At $\varphi=\Phi_{1}$ the energy density of $\chi$ particles equals the initial kinetic energy of the $\varphi$ field. At that point $\varphi$ stops and starts rolling back to SBP. It crosses this point practically with the same kinetic energy $\dot{\varphi} \simeq v t[22$ and triggers the second burst of particle production. The newly created particles are added to the total bath, which interact with $\varphi$ creating an even steeper potential for the latter. This way the oscillation amplitude rapidly decreases with each subsequent passage of SBP. The process continues until one of the two things happen. If the oscillation amplitude $\Phi$ decays bellow the non-adiabaticity region, the parametric resonance is no longer effective and particle production stops. Due to the tachyonic direction in our model, the particle production can be terminated by a different mechanism. As we saw in section $\mathrm{V}$, self-interactions can render the $\chi$ field too heavy for any further excitations. We called this process the "non-linear blocking". In some parameter space it halts any further particle production once it barely started. Which of the two mechanisms determines the end of the resonance depends on model parameters.

If the end of particle production is dictated by the first process, the trapping mechanism can also be responsible for an efficient reheating of the universe. This is an important advantage of the mechanism, as reheating in quintessential inflation scenarios is complicated, due to non-oscillatory potential (see refs. 27-29, 40, 45, for other possible alternatives). If, on the other hand, particle production is terminated by the non-linear blocking, then most likely reheating is much less efficient. However, this issue requires more detailed investigation.

Finally, we should mention that in this work we neglected the Goldstone boson $\theta$ of the $U(1)$ symmetry breaking. There are two possibilities, depending on a specific implementation. In ref. [21] it was suggested that $U(1)$ might be a global symmetry. In which case $\theta$ could constitute the QCD axionic dark matter in the Universe (once the potential of $\theta$ is lifted) [46-49]. In that case $f$ is limited to the "classical axion window", which is the range $10^{9-12} \mathrm{GeV}$ (assuming no fine-tuning of the misalignment angle). This is a particularly attractive possibility as it 
conforms to the main motivation of the quintessential inflation scenario by being minimal in its field content. If, on the other hand, we do not want to impose such a requirement and $U(1)$ is taken to be a local symmetry, then the $\theta$ field could be eaten by gauge bosons to make them heavy. In both cases, it is possible to avoid the 5 th force problems associated with light scalar fields.

\section{CONCLUSIONS AND DISCUSSION}

The paradigm of quintessential inflation can be very attractive to model the evolution of our Universe. The primary appeal of such models is that they do not introduce additional scalar fields beyond the inflaton. However, this minimalist approach also introduces certain difficulties. The main one being a mechanism of reheating the universe after inflation. In addition, with the usual quintessence models it shares the problem of the 5th force constraints, the need for the suppression of radiative corrections and explaining the absence of non-renormalisable terms. To solve those problems one might need to invoke other fields after all. However, this does not have to violate the principle of economy of quintessential inflation models. The additional field(s) can be the same that are already used in cosmology for different purposes.

This principle was applied in the model proposed in ref. [21], where the additional field is the same as used in QCD axion scenarios. The latter could explain the whole of dark matter in the Universe [48. If the interaction between the inflaton and the additional field is of the form in eq. (2), the problems mentioned above can be overcome. On the one hand, the interaction of this form induces a very rapid and efficient particle production, as the kinetic energy dominated inflaton zips through the symmetry breaking point (SBP). Such particles can stop the inflaton at sub-Planckian values and reheat the universe. On the other hand, the coupling makes the inflaton heavy in the vacuum, therefore preventing problems associated with the constraints from the 5th force experiments. Moreover, this scenario is not sensitive to the precise form of the potential in the quintessential tail. Only the hight of the potential is important.

The process of particle production is somewhat similar to the mechanism proposed in ref. 22. The crucial difference, however, is that the trapping field is self-interacting. These interactions lead to the non-linear evolution and change the efficiency of particle production and consequently the efficiency of the trapping mechanism and reheating.

In this work we study in detail how self-interactions affect the trapping. Within the Hartree approximation we develop the analytic formalism to account for the suppression of particle production. We also compute the particle number density in the region of non-linear blocking. In this region the self-interaction of the trapping field is so strong that it terminates particle production while the effective mass of the $\chi$ field is still tachyonic. The analytic results agree very well with numerical simulations as can be seen in figure 1 .

Using these analytic methods we can very efficiently explore the parameter space to compute the constraints. In ref. 21] the symmetry breaking scale $f$ was fixed. In this work we do not impose any bounds of $f$ (apart from observational constraints on cosmic string tension) and explore the full range of possibilities. Our results are provided in figure 2, where we show the parameter space for efficient trapping. One can see that this mechanism is effective for a very wide range of parameter values.

If the tachyonic trapping mechanism is indeed realised in Nature, dark energy would be indistinguishable from the cosmological constant. However, this mechanism suggests other potentially observable phenomena, such as the production of primordial gravitational waves as well as the formation of cosmic strings [50]. We leave the study of such possibilities for future publications.

\section{ACKNOWLEDGMENTS}

The work of M.K. and S.R. is supported by the Communidad de Madrid "Atracción de Talento investigador" Grant No. 2017-T1/TIC-5305 and MICINN (Spain) project PID2019-107394GB-I00. A. S. is supported by MICINN (Spain) grant PGC2018-094857-B-I00 and the Spanish Agencia Estatal de Investigación through the grant "IFT Centro de Excelencia Severo Ochoa" SEV-2016-0597 and CEX2020-001007-S.

\section{Appendix A: The Parabolic Cylinder Functions}

\section{The Properties of Parabolic Cylinder Functions}

In our analytic computations we make an extensive use of Parabolic Cylinder Functions (PCF). This is due to the fact that these functions form a complete set of solutions of eq. 21. However, even if the equation of motion 
of $\chi$ would be more complicated, PCF give a good approximation in the regions where the evolution of $\omega_{k}$ is nonadiabatic. Indeed, PCF are widely used in quantum mechanics precisely in this context: to find formulas connecting regimes with WKB approximate solutions. In this section we summarise the main properties of PCF that we use in the main text.

Consider the equation [51]

$$
y^{\prime \prime}+\omega_{\kappa}^{2} y=0,
$$

where

$$
\omega_{\kappa}^{2} \equiv \frac{1}{4} \tau^{2}-\kappa
$$

and primes denote derivatives with respect to the independent variable $\tau$. A generic solution of this equation can be written in terms of PCF as

$$
y=D_{1} W(\kappa, \tau)+D_{2} W(\kappa, \tau),
$$

where $D_{1,2}$ are integration constants. Equation A1 has a few equivalent forms related by redefinitions of $\kappa$ and $\tau$, but the above one is the easiest to apply to our setup.

At $\tau=0$ PCF reduce to

$$
W(\kappa, 0)=2^{-\frac{3}{4}}\left|\frac{\Gamma\left(\frac{1}{4}+\frac{1}{2} \mathrm{i} \kappa\right)}{\Gamma\left(\frac{3}{4}+\frac{1}{2} \mathrm{i} \kappa\right)}\right|^{\frac{1}{2}}
$$

and

$$
\frac{\mathrm{d} W(\kappa, 0)}{\mathrm{d} \tau}=-\frac{1}{2 W(\kappa, 0)},
$$

where $\Gamma$ is the gamma function. In the limit of large $\kappa$ this equation approaches

$$
W(\kappa, 0) \stackrel{\kappa \rightarrow \pm \infty}{\longrightarrow} \frac{1}{\sqrt{2}|\kappa|^{1 / 4}}
$$

To make the connection between PCF and the WKB approximate solutions we made use of several asymptotic forms of $W(\kappa, \tau)$. For $\tau^{2} \gg 4|\kappa|$ the $W$ function can be approximated at the lowest order in $\tau^{-2}$ by

$$
\begin{aligned}
W(\kappa, \tau) & \approx \sqrt{\frac{k}{2|\tau|}}\left(\mathrm{e}^{-\mathrm{i} \omega}+\mathrm{e}^{\mathrm{i} \omega}\right) \\
W(\kappa,-\tau) & \approx \frac{\mathrm{i}}{\sqrt{2 k|\tau|}}\left(\mathrm{e}^{-\mathrm{i} \omega}-\mathrm{e}^{\mathrm{i} \omega}\right)
\end{aligned}
$$

where

$$
\begin{aligned}
k & \equiv \sqrt{1+\mathrm{e}^{2 \pi \kappa}}-\mathrm{e}^{\pi \kappa} \\
\omega & \equiv \frac{1}{4} \tau^{2}-\frac{1}{2} \kappa \ln \tau^{2}+\frac{1}{4} \pi+\frac{1}{2} \phi_{2}
\end{aligned}
$$

and

$$
\phi_{2} \equiv \arg \left[\Gamma\left(\frac{1}{2}+\mathrm{i} \kappa\right)\right] .
$$

Notice that both functions, $W(\kappa, \tau)$ and $W(\kappa,-\tau)$, are real.

In the opposite regime, where $4|\kappa| \gg \tau^{2}$ the $W(\kappa, \tau)$ function can be approximated by

$$
\begin{aligned}
W(\kappa, \tau) & =W(\kappa, 0) \mathrm{e}^{-\sqrt{\kappa} \tau+v_{1}}, \\
W(\kappa,-\tau) & =W(\kappa, 0) \mathrm{e}^{\sqrt{\kappa} \tau+v_{2}},
\end{aligned}
$$

and

$$
v_{1,2} \simeq \pm \frac{\frac{2}{3}\left(\frac{1}{2} \tau\right)^{3}}{2 \sqrt{\kappa}}+\frac{\left(\frac{1}{2} \tau\right)^{2}}{(2 \sqrt{\kappa})^{2}} \pm \frac{\frac{1}{2} \tau+\frac{2}{5}\left(\frac{1}{2} \tau\right)^{5}}{(2 \sqrt{\kappa})^{3}}+\ldots
$$




\section{Relation to WKB Approximation}

For $\tau^{2} \gg|\kappa|$ and $\tau<0$ the $\omega_{\kappa}$ in eq. A2 changes adiabatically. Therefore, we can also find the solution of eq. (A1) using WKB approximation. Let us write this solution as

$$
y_{1} \simeq \frac{A_{1}}{\sqrt{2 \omega_{\kappa}}} \mathrm{e}^{-\mathrm{i} \int_{\tau_{0}}^{\tau} \omega_{\kappa} \mathrm{d} \tau}+\frac{A_{2}}{\sqrt{2 \omega_{\kappa}}} \mathrm{e}^{\mathrm{i} \int_{\tau_{0}}^{\tau} \omega_{\kappa} \mathrm{d} \tau} .
$$

$y_{1}$ is nothing else but the approximate expression of the exact solution in eq. A3. Indeed, using eqs. A7 and A8 we can find the connection formulas for the integration constants as

$$
\begin{aligned}
& D_{1}=\mathrm{i} \sqrt{\frac{k}{2}}\left[A_{1} \mathrm{e}^{-\mathrm{i}\left(\theta_{\kappa}+\varphi_{\kappa}\right)}-A_{2} \mathrm{e}^{\mathrm{i}\left(\theta_{\kappa}+\varphi_{\kappa}\right)}\right], \\
& D_{2}=\frac{1}{\sqrt{2 k}}\left[A_{1} \mathrm{e}^{-\mathrm{i}\left(\theta_{\kappa}+\varphi_{\kappa}\right)}+A_{2} \mathrm{e}^{\mathrm{i}\left(\theta_{\kappa}+\varphi_{\kappa}\right)}\right],
\end{aligned}
$$

where $k$ is defined in eq. A9, $\theta_{\kappa}$ is the phase accumulated from $\tau_{0}$ to $\tau_{-}$

$$
\theta_{\kappa} \equiv \int_{\tau_{0}}^{\tau_{-}} \omega_{\kappa} \mathrm{d} x
$$

and $\tau_{-}$is such that $\omega_{\kappa}^{2}\left(\tau<\tau_{-}\right)>0$, that is

$$
\tau_{-}= \begin{cases}0 & \text { if } \kappa \leq 0 \\ -\sqrt{2} \kappa & \text { if } \kappa>0\end{cases}
$$

The phase $\varphi_{\kappa}$ is defined as

$$
\varphi_{\kappa} \equiv \frac{1}{4} \pi+\frac{1}{2} \phi_{2}+\frac{1}{2} \kappa(1-\ln |\kappa|)
$$

where $\phi_{2}$ is given in eq. A11.

In the opposite regime, where $\tau^{2} \gg|\kappa|$ and $\tau>0$, we can derive similar connection formulas. Let us write the approximate WKB solution of the equation A1 as

where $\tau_{+}$is given by

$$
y_{3} \simeq \frac{C_{1}}{\sqrt{2 \omega_{\kappa}}} \mathrm{e}^{-\mathrm{i} \int_{\tau_{+}}^{\tau} \omega_{\kappa} \mathrm{d} \tau}+\frac{C_{2}}{\sqrt{2 \omega_{\kappa}}} \mathrm{e}^{\mathrm{i} \int_{\tau_{+}}^{\tau} \omega_{\kappa} \mathrm{d} \tau}
$$

$$
\tau_{+}=\left\{\begin{array}{ll}
0 & \text { if } \kappa \leq 0 \\
\sqrt{2} \kappa & \text { if } \kappa>0
\end{array},\right.
$$

Matching the approximate expression of eq. A3 one finds

$$
\begin{aligned}
C_{1} & =\frac{k D_{1}+\mathrm{i} D_{2}}{\sqrt{2 k}} \mathrm{e}^{-\mathrm{i} \varphi_{\kappa}}, \\
C_{2} & =\frac{k D_{1}-\mathrm{i} D_{2}}{\sqrt{2 k}} \mathrm{e}^{\mathrm{i} \varphi_{\kappa}} .
\end{aligned}
$$

Notice that using eqs. A16, A17 and (A23, A24 we can derive connection formulas

$$
\left(\begin{array}{l}
C_{1} \\
C_{2}
\end{array}\right)=\mathrm{i}\left(\begin{array}{cc}
\sqrt{1+\mathrm{e}^{2 \pi \kappa}} \mathrm{e}^{-\mathrm{i}\left(\theta_{\kappa}+2 \varphi_{\kappa}\right)} & \mathrm{e}^{\pi \kappa} \mathrm{e}^{\mathrm{i} \theta_{\kappa}} \\
-\mathrm{e}^{\pi \kappa} \mathrm{e}^{-\mathrm{i} \theta_{\kappa}} & -\sqrt{1+\mathrm{e}^{2 \pi \kappa}} \mathrm{e}^{\mathrm{i}\left(\theta_{\kappa}+2 \varphi_{\kappa}\right)}
\end{array}\right)\left(\begin{array}{c}
A_{2} \\
A_{2}
\end{array}\right)
$$

We can also find a WKB approximation of the solution in eq. A3 in the limit where $\kappa \gg \tau^{2}$. Let write the approximate solution of eq. A1 in this region as

$$
y_{2} \simeq \frac{B_{1}}{\sqrt{2\left|\omega_{\kappa}\right|}} \mathrm{e}^{-\int_{0}^{\tau}\left|\omega_{\kappa}\right| \mathrm{d} \tau}+\frac{B_{2}}{\sqrt{2\left|\omega_{\kappa}\right|}} \mathrm{e}^{\int_{0}^{\tau}\left|\omega_{\kappa}\right| \mathrm{d} \tau} .
$$

. We can expand $\int_{0}^{\tau}\left|\omega_{\kappa}\right| \mathrm{d} \tau$ and $\left|\omega_{\kappa}\right|^{-1 / 2}$ in terms of $\tau^{2} / 4 \kappa$ and match to the eq. A3 using the series expansion of PCF in eqs. A12 and A13. Note, that the series in eq. A14 contain terms that would correspond to higher order WKB approximation than provided in eq. A26. After the matching we find

$$
B_{1,2}=\sqrt{2} \kappa^{1 / 4} W(\kappa, 0) D_{1,2}
$$




\section{Appendix B: Laplace's Approximation}

To compute the integral in eq. (43) we used the Laplace's approximation [52. As the derivation of this particular integral involves a few steps, we recall them in this section.

The approximation can be summarised as

$$
I=\int_{0}^{\infty} \frac{\mathrm{e}^{C g(x)}}{f(x)} \mathrm{d} x \simeq \sqrt{\frac{2 \pi}{C\left|g^{\prime \prime}\left(x_{*}\right)\right|}} \frac{\mathrm{e}^{C g\left(x_{*}\right)}}{f\left(x_{*}\right)} .
$$

where $x_{*}$ is the value of $x$ such that $g\left(x_{*}\right)=\max (g)$ and primes denote derivatives with respect to $x$. The equality becomes exact in the limit $C \rightarrow \infty$.

In our case we need to compute the integral of the form

$$
I=\int_{0}^{\infty} \frac{x^{2}}{f(x)}\left(\sqrt{1+\mathrm{e}^{Q-x^{2}}}-1\right) \mathrm{d} x .
$$

To do that, let us first denote

$$
g(x) \equiv \ln \left[x^{2}\left(\sqrt{1+\mathrm{e}^{Q-x^{2}}}-1\right)\right]
$$

which has a maximum at

$$
Q=\ln \left[\frac{x_{*}^{2}-1}{\left(\frac{1}{2} x_{*}^{2}-1\right)^{2}}\right]+x_{*}^{2} .
$$

Unfortunately, we cannot solve this transcendental equation using elementary functions. But it suffices to notice that $x_{*}^{2} \in(1,2)$. In the main text we use an approximation such that for $Q \lesssim$ few. This corresponds to $x_{*}$ value

$$
x_{*}^{2} \simeq 1
$$

In this limit the approximate value of the integral is given by

$$
I \simeq 2^{-3 / 2} \sqrt{\pi} \frac{\mathrm{e}^{Q-1}}{f\left(x_{*}\right)} .
$$

In the opposite regime, for $Q \gg 1$, the largest contribution to the integral comes from $x$ values close to

$$
x_{*}^{2} \simeq 2
$$

and the approximate value of the integral is given by

$$
I \simeq 2 \sqrt{\pi} \frac{\mathrm{e}^{\frac{1}{2} Q-1}}{f\left(x_{*}\right)} .
$$

[1] S. Weinberg, Rev. Mod. Phys. 61, 1 (1989).

[2] N. Aghanim et al. (Planck), Astron. Astrophys. 641, A6 (2020), [Erratum: Astron.Astrophys. 652, C4 (2021)], arXiv:1807.06209 [astro-ph.CO].

[3] N. Weiss, Phys. Lett. B 197, 42 (1987).

[4] P. Peebles and B. Ratra, Astrophys. J. Lett. 325, L17 (1988).

[5] C. Wetterich, Nucl. Phys. B302, 668 (1988), arXiv:1711.03844 [hep-th]

[6] B. Ratra and P. J. E. Peebles, Phys. Rev. D37, 3406 (1988)

[7] P. G. Ferreira and M. Joyce, Phys. Rev. D 58, 023503 (1998), arXiv:astro-ph/9711102

[8] R. Caldwell, R. Dave, and P. J. Steinhardt, Phys. Rev. Lett. 80, 1582 (1998), arXiv:astro-ph/9708069

[9] P. J. E. Peebles and A. Vilenkin, Phys. Rev. D59, 063505 (1999), arXiv:astro-ph/9810509 [astro-ph].

[10] M. Peloso and F. Rosati, JHEP 12, 026 (1999), arXiv:hep-ph/9908271

[11] K. Dimopoulos and J. Valle, Astropart. Phys. 18, 287 (2002), arXiv:astro-ph/0111417.

[12] M. Wali Hossain, R. Myrzakulov, M. Sami, and E. N. Saridakis, Int. J. Mod. Phys. D24, 1530014 (2015), arXiv:1410.6100 [gr-qc] 
[13] C.-Q. Geng, M. W. Hossain, R. Myrzakulov, M. Sami, and E. N. Saridakis, Phys. Rev. D92, 023522 (2015), arXiv:1502.03597 [gr-qc]

[14] J. de Haro and L. A. Saló, Galaxies 9, 73 (2021), arXiv:2108.11144 [gr-qc].

[15] D. Bettoni and J. Rubio, (2021), arXiv:2112.11948 [astro-ph.CO]|

[16] S. M. Carroll, Phys. Rev. Lett. 81, 3067 (1998), arXiv:astro-ph/9806099 [astro-ph].

[17] C. F. Kolda and D. H. Lyth, Phys. Lett. B 458, 197 (1999), arXiv:hep-ph/9811375

[18] E. V. Linder, Phys. Rev. D 91, 123012 (2015), arXiv:1505.00815 [astro-ph.CO]

[19] K. Dimopoulos and C. Owen, JCAP 1706, 027 (2017), arXiv:1703.00305 [gr-qc].

[20] L. A. Saló, D. Benisty, E. I. Guendelman, and J. de Haro, Phys. Rev. D 103, 123535 (2021), arXiv:2103.07892 [astroph.CO].

[21] K. Dimopoulos, M. Karčiauskas, and C. Owen, Phys. Rev. D100, 083530 (2019), arXiv:1907.04676 [hep-ph].

[22] L. Kofman, A. D. Linde, X. Liu, A. Maloney, L. McAllister, and E. Silverstein, JHEP 05, 030 (2004), arXiv:hepth/0403001 [hep-th].

[23] W. L. Freedman et al., (2019), 10.3847/1538-4357/ab2f73, arXiv:1907.05922 [astro-ph.CO]

[24] D. W. Pesce et al., Astrophys. J. Lett. 891, L1 (2020), arXiv:2001.09213 [astro-ph.CO].

[25] L. Verde, T. Treu, and A. G. Riess, Nature Astron. 3, 891 (2019), arXiv:1907.10625 [astro-ph.CO].

[26] A. Banerjee, H. Cai, L. Heisenberg, E. O. Colgáin, M. M. Sheikh-Jabbari, and T. Yang, Phys. Rev. D 103, L081305 (2021), arXiv:2006.00244 [astro-ph.CO].

[27] A. H. Campos, H. C. Reis, and R. Rosenfeld, Phys. Lett. B575, 151 (2003), arXiv:hep-ph/0210152 [hep-ph]

[28] A. Agarwal, R. Myrzakulov, M. Sami, and N. K. Singh, Phys. Lett. B 770, 200 (2017), arXiv:1708.00156 [gr-qc].

[29] K. Dimopoulos, L. D. Wood, and C. Owen, Phys. Rev. D97, 063525 (2018), arXiv:1712.01760 [astro-ph.CO],

[30] B. Spokoiny, Phys. Lett. B315, 40 (1993), arXiv:gr-qc/9306008 [gr-qc].

[31] M. Joyce, Phys. Rev. D 55, 1875 (1997), arXiv:hep-ph/9606223.

[32] L. Kofman, A. Linde, and A. A. Starobinsky, Phys. Rev. D56, 3258 (1997), arXiv:hep-ph/9704452 [hep-ph].

[33] J. F. Dufaux, G. N. Felder, L. Kofman, M. Peloso, and D. Podolsky, JCAP 0607, 006 (2006), arXiv:hep-ph/0602144 [hep-ph].

[34] E. Merzbacher, Quantum mechanics (Wiley, New York, 1998).

[35] R. Brustein, S. P. De Alwis, and E. G. Novak, Phys. Rev. D 68, 023517 (2003), arXiv:hep-th/0205042

[36] A. Lazanu and P. Shellard, JCAP 02, 024 (2015), arXiv:1410.5046 [astro-ph.CO].

[37] J. Lizarraga, J. Urrestilla, D. Daverio, M. Hindmarsh, and M. Kunz, JCAP 10, 042 (2016), arXiv:1609.03386 [astroph.CO]

[38] P. Ade et al. (Planck), Astron. Astrophys. 571, A25 (2014), arXiv:1303.5085 [astro-ph.CO]

[39] D. Bettoni and J. Rubio, JCAP 01, 002 (2020), arXiv:1911.03484 [astro-ph.CO].

[40] K. Dimopoulos and T. Markkanen, JCAP 1806, 021 (2018), arXiv:1803.07399 [gr-qc]

[41] T. Opferkuch, P. Schwaller, and B. A. Stefanek, JCAP 07, 016 (2019), arXiv:1905.06823 [gr-qc]

[42] D. Bettoni, A. Lopez-Eiguren, and J. Rubio, (2021), arXiv:2107.09671 [hep-ph]

[43] K. Dimopoulos and D. H. Lyth, Phys.Rev. D69, 123509 (2004), arXiv:hep-ph/0209180 [hep-ph]

[44] K. Dimopoulos and G. Lazarides, Phys. Rev. D 73, 023525 (2006), arXiv:hep-ph/0511310.

[45] G. B. F. Lima and R. O. Ramos, Phys. Rev. D 100, 123529 (2019) arXiv:1910.05185 [astro-ph.CO].

[46] A. Ringwald, Phys. Dark Univ. 1, 116 (2012), arXiv:1210.5081 [hep-ph]

[47] M. Kawasaki and K. Nakayama, Ann. Rev. Nucl. Part. Sci. 63, 69 (2013), arXiv:1301.1123 [hep-ph],

[48] H. Baer, K.-Y. Choi, J. E. Kim, and L. Roszkowski, Phys. Rept. 555, 1 (2015) arXiv:1407.0017 [hep-ph].

[49] D. J. E. Marsh, Phys. Rept. 643, 1 (2016), arXiv:1510.07633 [astro-ph.CO]|

[50] D. Bettoni, G. Domènech, and J. Rubio, JCAP 02, 034 (2019), arXiv:1810.11117 [astro-ph.CO].

[51] M. Abramowitz and I. A. Stegun, Handbook of Mathematical Functions with Formulas, Graphs, and Mathematical Tables, ninth dover printing, tenth gpo printing ed. (Dover, New York, 1964).

[52] R. W. Butler, Saddlepoint approximations with applications (Cambridge University Press, New York, 2007). 